Correction to "Cyclic Division Algebras", by Louis Halle Rowen, Israel Journal of Mathematics, Vol. 41, No. 3, 1982, pp. 213-234.

Tignol has spotted two errors in the proof of [1, Theorem 6]. The proof given below requires a more careful analysis, based on the original idea but taking into account that the number of roots of unity available at each stage changes.

Fix any power $f$ of $p$, where $f \geqq p$ for $p$ odd and $f \geqq 4$ for $p=2$. Take $\hat{K}=Q\left(\zeta_{f}\right)\left(\mu_{1}, \cdots, \mu_{u}\right)$ where $u=f n / p$ and let $K$ be the fixed subfield under $\sigma^{n}$ (where $\sigma$-permutes the $\mu_{i}$ cyclically). Then $R=\left(K, \sigma, \zeta_{f}\right)$ is a division algebra by Brauer's Theorem, which we claim has exponent $p$. (Indeed we argue as in Example 3. Let $K_{1}$ be the fixed subfield of $K$ under $\sigma^{n / p}$. Then $\sigma^{n / p}(x)=\zeta_{f} x$ for some $x$ in $\hat{K}$ so $\sigma(x)=a_{1} x$ for some $a_{1}$ in $K_{1}$; hence $\zeta_{f}=\sigma^{n / p-1}\left(a_{1}\right) \cdots a_{1}$ and $\zeta_{f}^{p}=N\left(a_{1}\right)$ proving $\exp (R) \leqq p$; equality holds since $\exp (R) \neq 1$.)

Form $R^{\prime}$ as in $\$ 1$ by taking $m=p=q$. Then $R^{\prime}$ has degree $n$ and exponent $p$ by [1, Remark 6$]$, and this is the example to be used for [1, Theorem 6$]$ and $[1$, Theorem 8]. (Note for $f=p$ we have $u=n$, which provided the example originally considered.)

Proof of [1, Theorem 6]. Suppose $R^{\prime}$ is a crossed product with respect to the split Galois group of exponent $p$. By Example 2, $R^{\prime}$ has a commutative $p$-central set of order $n$. Thus, by Remark 7, R has a commutative $p$-central set $S$ of order $n$ all of whose elements are in $R_{0} k^{i} z^{i}$ for various $i, j>p$, where we recall $K=K_{0}(k)$, and $R_{0}$ is the subalgebra of $R$ generated by $K_{0}$ and $z^{p}$.

We need some more notation. For any given $d$ let $c=n / p^{d+1}$ and let $K_{d}$ denote the fixed subfield of $K$ under $\sigma^{c}$; let $R_{d}$ be the subalgebra of $R$ generated by $K_{d}$ and $z^{p}$. Then $z^{c} \in Z\left(R_{d}\right)$ and is thus identified in $R_{d}$ as a primitive $p^{d+1}$-root of $\zeta_{f}$.

Given a commutative $p$-central set $S_{d-1}$ of elements $s_{t}=r_{t} z^{j_{t}}$ for suitable $r_{t}$ in $R_{d-1}$, let $\zeta=z^{p c}$, a primitive $p^{d}$-root of $\zeta_{f}$. Put $P=Q\left(\zeta_{f}\right)$ and $H=P\left[\mu_{1}, \cdots, \mu_{u}\right]$. Writing $r_{i}=\sum_{i=0}^{c-1} x_{i,} z^{p i}$ for suitable $x_{i, t}$ in $K_{d-1}(\zeta)$ and multiplying through by a suitable element of $F$ we may assume all $x_{i, t} \in H$. Put $V=\sum_{j=1}^{u} P \mu_{j}$ and write $V=\bigoplus_{i=1}^{i} V_{i}$, a finite direct sum of $e$ irreducible $\sigma$-submodules. For each $\alpha=\left(\alpha_{1}, \cdots, \alpha_{e}\right)$ write $V_{\alpha}=\prod_{i=1}^{e} V_{i}^{\alpha_{i}}$. Note the $V_{\alpha}$ are homogeneous in total degree in the $\mu_{j}$, so an easy dimension counting argument shows $H=\bigoplus_{\alpha} V_{\alpha}$ is graded as $\sigma$-module, and we order the $V_{\alpha}$ according to the lexicographic order of $\alpha$. Let $V_{\alpha_{t}}$ denote the leading component of all $x_{i, t}$ appearing in $r_{t}$, let $x_{t, t}^{t}$ denote the $V_{\alpha_{t}}$-component of $x_{i, t}$ (possibly 0), let $r_{t}^{\prime}=\sum_{i=0}^{c-1} x_{i, t}^{\prime}, z^{p i}$ and $s_{t}^{\prime}=r_{t}^{\prime} z^{i}$. Then the $s_{\imath}^{\prime}$ form a new commutative $p$-central set $S_{d-1}^{\prime}$ of which we claim 
$\left|S_{d-1}\right| / p$ elements have $r_{t}^{\prime}$ in $R_{d}$; this subset of $\left|S_{d-1}\right| / p$ elements from $S_{d-1}^{\prime}$ will be denoted as $S_{d}$. (Note that each $r_{t}^{\prime}$ is fixed by $\sigma^{p c}$, where $\sigma$ acts naturally on $H$ by setting $\sigma(\zeta)=\zeta$.)

The passage from $S_{d-1}$ to $S_{d}$ will be called Brauer's degree reduction argument, based on Jacobson's exposition of Brauer, and the above claim can be proved by proving the following stronger assertion:

$$
z^{c} r_{t}^{\prime} z^{-c}=\zeta(t) r_{t}^{\prime} \quad \text { where } \zeta(t) \text { is a suitable } p \text { th-root of } 1 .
$$

To see this, first note $\sigma^{c}$ induces a transformation on $V_{i}$ whose order divides $p^{d+1}$, so $\sigma^{c}\left(w_{i}\right)=\zeta^{(i)} w_{i}$ for some nonzero $w_{i} \in V_{i}$ and some power $\zeta^{(i)}$ of $\zeta$. The characteristic subspace of $\zeta^{(i)}$ under $\sigma^{c}$ is a nonzero $\sigma$-submodule of $V_{i}$ (since $\sigma^{c}(w)=\zeta^{(i)} w$ implies $\left.\sigma^{c}(\sigma w)=\sigma\left(\sigma^{c} w\right)=\sigma\left(\zeta^{(i)} w\right)=\zeta^{(i)} \sigma(w)\right)$ and is thus all of $V_{i}$, i.e. $\sigma^{c}(w)=\zeta^{(i)} w$ for all $w$ in $V_{i}$. Hence for each $\alpha=\left(\alpha_{1}, \alpha_{2}, \cdots, \alpha_{e}\right)$ there is some power $\zeta^{(\alpha)}$ of $\zeta$ with $\sigma^{c}(w)=\zeta^{(\alpha)} w$ for each $w$ in $V_{\alpha}$. Writing $\zeta(t)$ for $\zeta^{\left(\alpha_{\gamma}\right)}$ note that $\zeta(t)^{p}=1$ (since $\sigma^{p c}\left(x_{i, t}\right)=x_{i, t}$ by hypothesis), and

$$
z^{c} r_{t}^{\prime} z^{-c}=z^{c}\left(\sum_{i=0}^{c-1} x_{i, t}^{\prime} z^{p i}\right) z^{-c}=\sum_{i=0}^{c-1} z^{c} x_{i, t}^{\prime} z^{-c} z^{p i}=\sum \zeta(t) x_{i, t}^{\prime} z^{p i}=\zeta(t) r_{t}^{\prime},
$$

proving the claim.

For example, we see from the first paragraph that we could take $\left|S_{0}\right|=n / p$ yielding $\left|S_{d}\right|=c$ for each $d$. We need some more observations about this reduction argument.

REMARK. If $s \in S_{d-1}$ and $s^{\prime} \in F z^{n / p}$ then $s \in F z^{n / p}$. (Indeed $s=\left(\alpha z^{n / p}+r\right) z^{j}$ where $\alpha \in H \cap F$ is the leading component, $r \in H \cap R_{d-1}$, and $j<p$. Hence $j=0$; since $r$ commutes with $z^{n / p}$ we get $s^{p}=\alpha^{p} z^{n}+p \alpha^{p-1} z^{(p-1) n / p} r+\cdots \in F$. Hence the leading component of $p \alpha^{p-1} z^{(p-1) n / p} r$ is in $F$, so the leading component of $r$ is in $F z^{n / p}$; working inductively yields $r \in F z^{n / p}$.)

REMARK. If $s \in S_{d-1}$ and $s^{\prime} \in F k^{\prime \prime} z^{\text {in/p }}$ where $k^{\prime \prime}$ is any element of $K$ such that $\sigma\left(k^{\prime \prime}\right)=\zeta_{p} k^{\prime \prime}$, then $s \in F k^{\prime \prime} z^{i n / p}$. (Indeed $k^{\prime \prime}$ commutes with $z^{p}$ so the argument of the previous remark applies.)

Iterating each remark over all $d$, we may assume that if $z^{n / p}$ or $k^{\prime \prime} z^{\text {in/p }}$ appears in any $S_{d}$ then it already appears in $S_{0}$ (and thus in $S$ ). For the remainder of the proof fix $d=\log _{p} n-2$, i.e., $p^{d+2}=n$. Then $c=p$ and $R_{d}=K_{d}\left(z^{p}\right)$ is a field in which $z^{p}$ is identified with $\zeta_{u}$; hence $R_{d}$ cannot have a $p$-central set of more than $p^{2}$ elements. Also $K_{d}=F\left(k_{d}\right)$ for suitable $k_{d}$ where $\sigma\left(k_{d}\right)=\zeta_{p} k_{d}$, and $R_{d-2}$ is a division ring of degree $p^{2}$ whose center contains $z^{p^{3}}\left(u / p^{2}\right.$-root of 1$)$. 
CLATM 1. If $s \in S_{d-2} \cap R_{d-2}$ then $s^{\prime} \in R_{d-1}$ (notation as before); in particular if $S_{d-2} \subseteq R_{d-2}$ then $S_{d-2}^{\prime} \subseteq R_{d-1}$ and we may take $S_{d-1}$ to be all of $S_{d-2}^{\prime}$.

Proof OF Claim 1. Otherwise $\sigma^{p^{2}}\left(s^{\prime}\right)=\zeta_{p} s^{\prime}$ for some $p$-th root $\zeta_{p}$ of 1 . Hence $z^{p^{2}}$ and $s^{\prime}$ generate a cyclic central subalgebra of $R_{d-2}$ having degree $p$, whose centralizer thus also has degree $p$. Conclude as in Claim 1 of the original proof.

Remark. $S_{d} \subseteq R_{d}$. Indeed otherwise we have $r z^{j}$ in $S_{d}$ with $r \in R_{d}$ and $j \neq 0$. Then $r \sigma(r) \cdots \sigma^{p-1}(r) z^{p j} \in F$ contrary to Proposition 6 , where the notation $L_{1}, L$, and $h$ of Proposition 6 are replaced here respectively by $R_{d}=$ $K_{d}\left(z^{p}\right)=K_{d}\left(\zeta_{u}\right), K_{d}$ and $r$ (i.e. $e=u$ in Proposition 6).

Clalm 2. We may assume $S$ contains $z^{n / p}$ and $k_{d}$.

Proof of Claim 2. We showed $\left|S_{d}\right| \geqq p$ and $S_{d} \subseteq R_{d} \approx K_{d}\left(\zeta_{u}\right)$, so we may assume $S_{d}$ contains $z^{n / p}$ or $k_{d} z^{i n / p}$ for some $i$. By the above remarks we may assume $S$ contains one of these elements; we need to prove $S$ contains both of them or, equivalently, $\left|S_{d}\right|=p^{2}$.

If $z^{n / p} \in S$ then each $s_{t}$ in $S$ commutes with $z^{n / p}$ and thus has suitable form $r_{t} z^{i_{k}}$ for $r_{t}$ in $R_{0}$, i.e., $\left|S_{0}\right|=n$; then the Brauer reduction argument yields $\left|S_{d}\right|=p^{2}$ and we are done. If $k_{d} z^{i n / p} \in S$ then each $s_{t} \in R_{0}\left(k z^{i}\right)^{i}$ for some $i_{t}$, so we could find $S_{0} \subseteq R_{0}$. Then $S_{d-2} \subset R_{d-2}$ so by Claim 1 we may take $\left|S_{d-1}\right|=p^{3}$ and so $\left|S_{d}\right|=p^{2}$, proving Claim 2 .

But now we know $S$ is centralized by $z^{n / p}$ and $k_{d}$, implying $S \subseteq R_{0}$. Hence we may take $S_{0}=S$ and $\left|S_{0}\right|=n$, so $\left|S_{d-2}\right|=p^{4}$ and $S_{d-2} \subset R_{d-2}$, implying $\left|S_{d-1}\right|=p^{4}$ by Claim 1 and $\left|S_{d}\right|=p^{3}$, contrary to $S_{d} \subset R_{d}$.

Q.E.D.

\section{Added in proof}

Unfortunately this argument opens up another gap, namely, letting $T_{d}$ denote the subalgebra of $R$ generated by $K_{d}$ and $z$ (so that $R_{d}$ is the centralizer of $z^{p}$ in $T_{d}$ ) and $F_{d}=Z\left(T_{d}\right)=F\left(z^{c}\right)$, we do not necessarily have the $s_{\text {t }}^{\prime}$ independent over $F_{d}$ (although each $\left(s_{i}^{\prime}\right)^{p} \in Z\left(R_{d}\right)$ ). To assure this we must make a further modification. Write $S_{d-1}=s_{1}, \cdots, s_{u}$ and $K_{d-1}=K_{d}\left(k_{d-1}\right)$ with $\sigma^{c}\left(k_{d-1}\right)=\zeta_{p} k_{d-1}$. Taking leading components (denoted as ') we may assume $k_{d-1}$ is homogeneous. We note the following for all $t$ :

(i) If $s_{t} \notin F_{d-1}$ then $s_{t}^{\prime} \notin F_{d-1}$ (for if $s_{t} r=\zeta_{p} r s_{t}$ then $s_{t}^{\prime} r^{\prime} \neq r^{\prime} s_{t}^{\prime}$ ).

(ii) $\left(s_{t}^{t}\right)^{p} \in F_{d-1}$ (for $s_{t}^{p} k_{d-1}=k_{d-1} s_{t}^{p}$, implying $\left.\left(s_{t}^{t}\right)^{p} k_{d-1}=k_{d-1}\left(s_{t}^{\prime}\right)^{p}\right)$.

(iii) If $s_{t} \in F_{m}$ for any $m \geqq d$ then $s_{t} \in F_{d-1} z^{c j}$ for some $j$ (since $s_{t}^{p} \in F_{d-1}$ and $F_{m}$ is cyclic over $F_{d-1}$ ). Likewise by (ii), if $s_{,}^{\prime} \in F_{m}$ then $s_{t}^{\prime} \in F_{d-1} z^{c j}$ for some $j$. 
(iv) If $s_{t}^{\prime} \in F_{d+1}$ then $s_{t} \in F_{d}$. Indeed $s_{t}^{\prime}=\alpha z^{\text {cj }}$ for some $\alpha \in F_{d-1}$ by (iii), so $s_{t}=\alpha z^{c i}+r$ for $r \in H \cap R_{d-1}$ of lower order. As in the Remark above, we get $r \in F_{d-1} z^{c j}$ so $s_{t} \in F_{d-1} z^{c j} \subset F_{d}$.

(v) Analogously, if $s_{t}^{\prime} \in F_{d+1} k^{\prime \prime}$ where $\sigma k^{\prime \prime}=\zeta_{p} k^{\prime \prime}$ then $s_{t} \in F_{d} k^{\prime \prime}$.

Now as in the argument in the original correction $z^{n / p} \in S$. Thus $\left|S_{0}\right|=n$ so we can replace $S_{0}$ by a set of $n / p$ elements which are $F\left(z^{n / p}\right)$-independent. Now we finally can claim $S_{d}$ is $p$-central in $T_{d}$. This is clear by induction unless say $F_{d}\left(s_{1}^{\prime}\right)=F_{d}\left(s_{2}^{\prime}\right)$, so $s_{2}^{\prime} \in F_{d} s_{1}^{\prime j}$ for some $j$ implying $s_{1}^{\prime} s_{2}^{-1} \in F_{d}$ by (iv) which implies $S_{0}$ has two elements $x_{1}, x_{2}$ with $x_{1}^{j} x_{2}^{-1} \in F_{0}=F\left(z^{n / p}\right)$, contrary to assumption.

\section{REFERENCE}

1. L. H. Rowen, Cyclic division algebras, Isr. J. Math. 41 (1982), 213-234.

\section{Department of MATHEMatics}

BAR ILAN UNIVERSITY

RAMAT GaN, IsRAEL 